This is the Accepted Author Manuscript of the publication

Cross-validation of biomarkers to early differential diagnosis and prognosis of dementia in a clinical setting

Perani D, Cerami C, Caminiti SP, Santangelo R, Coppi E, Ferrari L, Pinto P, Passerini G, Falini A, Iannaccone S, Cappa SF, Comi G, Gianolli L, Magnani G.

"Corresponding author:

Prof. Daniela Perani, MD

Vita-Salute San Raffaele University,

Nuclear Medicine Center, San Raffaele Hospital,

Division of Neuroscience, San Raffaele Scientific Institute

Via Olgettina 60, Milan 20132, Italy.

Tel: +390226432224 or 26432223

Fax: +390226415202 .

E-mail address: perani.daniela@hsr.it (D. Perani).

Published in: European Journal of Nuclear Medicine and Molecular Imaging March 2016, Volume 43, Issue 3, pp 499-508

First online: 04 September 2015

Doi: $10.1007 / \mathrm{s} 00259-015-3170-\mathrm{y}$

The final publication is available at http://link.springer.com/article/10.1007\%2Fs00259-015-3170-y

(C) 2016. This manuscript version is made available under the CC-BY-NC-ND 4.0 license http://creativecommons.org/licenses/by-nc-nd/4.0/ 


\section{Cross-validation of biomarkers to early differential diagnosis and prognosis of dementia in a clinical setting}

Daniela Perani $^{\mathrm{a}, \mathrm{b}, \mathrm{c}^{*}}$, Chiara Cerami ${ }^{\mathrm{a}, \mathrm{b}, \mathrm{d}}$, Silvia Paola Caminiti ${ }^{\mathrm{a}, \mathrm{b}}$, Roberto Santangelo ${ }^{\mathrm{e}}$, Elisabetta Coppi $^{\mathrm{e}}$, Laura Ferrari ${ }^{\mathrm{e}}$, Patrizia Pinto ${ }^{\mathrm{f}}$, Gabriella Passerini ${ }^{\mathrm{g}}$, Andrea Falini ${ }^{\mathrm{a}, \mathrm{b}, \mathrm{h}}$, Sandro

Iannaccone $^{\mathrm{d}}$, Stefano Francesco Cappa ${ }^{\mathrm{b}, \mathrm{i}}$, Giancarlo Comi ${ }^{\mathrm{a}, \mathrm{e}}$, Luigi Gianolli ${ }^{\mathrm{c}}$, Giuseppe Magnani ${ }^{\mathrm{e}}$
a. Vita-Salute San Raffaele University, Via Olgettina, 58, 20132 Milan, Italy
b. Division of Neuroscience, San Raffaele Scientific Institute, Via Olgettina, 58, 20132 Milan, Italy
c. Nuclear Medicine Unit, San Raffaele Hospital, Via Olgettina, 60, 20132 Milan, Italy
d. Clinical Neuroscience Department, San Raffaele Hospital, Via Olgettina, 60, 20132 Milan, Italy
e. Department of Neurology, San Raffaele Hospital, Via Olgettina, 60, 20132 Milan, Italy
f. Department of Neurology, Papa Giovanni XXIII Hospital, Piazza OMS, 1, 24127 Bergamo, Italy
g. Servizio di Medicina di Laboratorio OSR, Via Olgettina, 60, 20132 Milan, Italy
h. CERMAC-Department of Neuroradiology, San Raffaele Hospital, Via Olgettina, 60, 20132 Milan, Italy
i. IUSS Pavia Piazza della Vittoria, 15, 27100 Pavia, Italy

Daniela Perani, e-mail: perani.daniela@hsr.it

ChiaraCerami, e-mail: cerami.chiara@hsr.it

Silvia Paola Caminiti, e-mail: caminiti.silviapaola@hsr.it

Roberto Santangelo, e-mail: santangelo.roberto@hsr.it

ElisabettaCoppi, e-mail: coppi.elisabetta@hsr.it

Laura Ferrari, e-mail: ferrari.laura@hsr.it

Patrizia Pinto, e-mail: ppinto@hpg23.it

Gabriella Passerini, e-mail: passerini.gabriella@hsr.it

Andrea Falini, e-mail: falini.andrea@hsr.it

Sandro Iannaccone, e-mail: iannaccone.sandro@hsr.it

Stefano Francesco Cappa, e-mail: stefano.cappa@iusspavia.it

GiancarloComi, e-mail: comi.giancarlo@hsr.it

Luigi Gianolli, e-mail: gianolli.luigi@hsr.it

Giuseppe Magnani, e-mail: magnani.giuseppe@hsr.it

\section{"Corresponding author:}

\section{Prof. Daniela Perani, MD}

Vita-Salute San Raffaele University,

Nuclear Medicine Center, San Raffaele Hospital,

Division of Neuroscience, San Raffaele Scientific Institute

Via Olgettina 60, Milan 20132, Italy.

Tel: +390226432224 or 26432223

Fax: +390226415202 .

E-mail address: perani.daniela@hsr.it (D. Perani). 


\title{
ABSTRACT
}

Purpose: The aim of this study was to evaluate the supportive role of molecular and structural biomarkers (CSF, FDG-PET and MRI) to the early differential diagnosis of dementia in a large sample of neurodegenerative dementia cases, and to the risk of disease progression in mild cognitive impairment (MCI). Methods: We evaluated the supportive role of CSF A $\beta_{42}$, t-Tau, pTau, conventional brain MRI and visual assessment of FDG-PET SPM t-map to early dementia diagnosis and MCI progression evaluation. Results: Diagnosis based on molecular biomarkers showed the better fit with the final diagnosis at a long follow-up. FDG-PET SPM t-maps had the highest diagnostic accuracy in $\mathrm{AD}$ and in the differential diagnosis of non- $\mathrm{AD}$ dementias. The $\mathrm{p}-$ tau/A $\beta_{42}$ ratio was the only CSF biomarker providing a significant classification rate for $\mathrm{AD}$. An AD positive metabolic pattern as shown by FDG-PET SPM was the best predictor for conversion to AD in the MCI group. Conclusions: In this clinical setting, FDG-PET SPM t-maps and p$\operatorname{tau} / \mathrm{A} \beta_{42}$ ratio improved the clinical diagnostic accuracy, supporting the importance of these biomarkers in the emerging diagnostic criteria for AD dementia. Noteworthy, FDG-PET using SPM t-maps had the highest predictive value by identifying hypometabolic patterns for different neurodegenerative dementias and normal brain metabolism in MCI, confirming also its crucial exclusionary role.

\section{Keywords}

Alzheimer's Disease, Mild Cognitive Impairment, Biomarkers, Dementia diagnosis, clinical setting.

\author{
Abbreviations \\ $A D=$ Alzheimer's disease \\ $D L B=$ Dementia with Lewy bodies \\ $F T L D=$ Frontotemporal Lobar Dementia \\ $M C I=$ Mild Cognitive Impairment \\ $C S F=$ Cerebrospinal Fluid \\ FDG-PET = Fluorodeoxyglucose- Positron Emission Tomography
}


$M R I=$ Magnetic Resonance Imaging

AUC $=$ Area Under the Curve

$L R=$ Likelihood Ratio

Sens $=$ Sensitivity

Spec $=$ Specificity 


\section{INTRODUCTION}

The World Alzheimer Report (2011) underlined that only 20 to $50 \%$ of dementia cases are early recognized [1]. The so called "treatment gap" between the early stages of pathology and a correct diagnosis leads an increase in the public costs and may be related to poor efficacy of treatments [2]. Moreover, different studies found that between $12 \%$ and $23 \%$ of diagnoses of Alzheimer's disease (AD) are not confirmed at autopsy ("misdiagnosed") [3]. These misdiagnoses may be due to the fact that different pathological conditions mimic symptoms of AD. Although diagnostic criteria of dementia are constantly improving (e.g., [4-10]), several challenges in terms of differential and early diagnosis of dementias remain in a clinical setting. Thus, the validation of dementia biomarkers supporting a more certain early and differential diagnosis has become urgent [11].

In the case of $\mathrm{AD}$, there is evidence of distinctive topographical and pathological markers for symptomatic, prodromal and also preclinical AD condition [12-19]. Both the National Institute on Aging-Alzheimer's Association workgroup (NIA-AA) and the International Working Group (IWG) proposed a significant revision of guidelines for preclinical/asymptomatic, prodromal (mild cognitive impairment (MCI) due to AD), and symptomatic AD conditions $[4,5,10,20]$, centring the diagnosis on the supportive use of biomarkers.

Cerebrospinal fluid (CSF) levels of $A \beta_{42}$, total tau (t-Tau) and phosphorylated tau (p-Tau) proteins have been extensively investigated as potential in vivo markers of AD pathology, in the typical and atypical AD conditions [21,22], as well as in prodromal AD [15]. Low CSF A $\beta_{42}$ levels in combination with high t-Tau/p-Tau showed high sensitivity and specificity in AD and high predictive value for progression of MCI to AD [23]. Since variations in the procedures may account for different accuracy [24], worldwide standard operating procedures have been suggested [24].

Structural MRI has been recognized as a useful measure of cerebral atrophy of either whole brain or, more specifically, of perirhinal and entorhinal cortices in AD [16]. Medial temporal 
lobe atrophy however, is not specific for AD condition, since it may also be present both in other neurological conditions [25-27] and in advancing age [28]. For this reasons, it has a limited value in differential diagnosis of $\mathrm{AD}$ condition [16].

FDG-PET provides in vivo information about the distribution of synaptic dysfunction in dementia and represents the most proximal biomarker associated with the neuronal dysfunction of $\mathrm{AD}$, closely related to its clinical presentation $[29,30]$. In the last twenty years, FDG-PET imaging earned increasing supportive role in the diagnostic algorithm of AD [31]. In addition, research studies on a memory clinic setting showed that FDG-PET has added value over the standard diagnostic work-up, influencing the final diagnosis $[32,33]$. This is especially true when prior diagnostic confidence is low [34]. Since neurodegenerative dementias present selective neuronal vulnerabilities, according to which specific neuronal population dies and others are resistant to neurodegeneration, specific topographical patterns of cerebral hypometabolism may be detected also at a single-subject level $[14,33,35]$.

The simple use of biomarkers in the diagnostic algorithm is not sufficient alone to guarantee a greater accuracy. Their accuracy depends on the type (e.g., amyloid PET imaging, FDG-PET or MRI) as well as on the measurement method [36]. Semi-quantitative or quantitative assessment and standard operating procedures are strongly suggested by scientific societies as key to a welladvised use of biomarkers in research, clinical settings and trials[37]. In addition, the combined use of different biomarkers and the standardized procedures are expected to facilitate the diagnostic process at the single-subject level [38-40].

Previous studies have provided evidence for the role of biomarkers in dementia diagnosis and in risk progression to dementia in MCI using the direct comparison of the three biomarkers (i.e., CSF, MRI and FDG-PET) [41-46]. These studies reported some discrepancies in their effective role that might depend on different aspects, such as the included population and possible clinical misdiagnoses, the differential role of biomarkers during the disease course, and, crucially, on the biomarker measurement methods [47]. Accuracy of imaging biomarkers can be indeed very 
different whether based on parametric approaches or on visual inspection that greatly depends on the observer's experience and lacks of a clear cut-off between normal and pathological findings [36].

In this study, we aimed at evaluating the role of CSF measures, MRI and FDG-PET biomarkers as available in a routine clinical setting for the early differential diagnosis of AD and for prediction of progression to dementia in MCI subjects. In particular, we evaluated the role of CSF $A \beta_{42}$, t-Tau and p-Tau proteins levels, of atrophy on visual assessment of conventional MRI, and of visual assessment of FDG-PET Statistical Parametrical Mapping (SPM) t-maps $[33,48]$ in a large sample of subjects attending a memory clinic. We assessed the power of each biomarker and their combined use for the early differential diagnosis of dementia and their predictive value in the progression to dementia in MCI subjects. Given the previous evidence of a very high diagnostic accuracy of our new validated procedure for the routine assessment of FDG-PET imaging in clinical setting $[33,48]$, we predicted that this biomarker would provide superior performance compared to the other biomarkers.

\section{MATERIAL AND METHODS}

\subsection{Subjects}

We retrospectively collected information about 86 early dementia (i.e., AD, frontotemporal lobar degeneration-FTLD and dementia with Lewy bodies-DLB) patients and 35 MCI subjects. All individuals were referred to the memory clinics of the San Raffaele Hospital (Milan, Italy) with memory or other cognitive impairments during the period between 2009 and 2012. At referral, they were evaluated by experienced behavioral neurologists and neuropsychologists. Clinical evaluation included a structured clinical interview, a full neurological examination, and a standard neuropsychological evaluation. See Perani et al. [33] for details on the neuropsychological battery used in our clinical setting. All patients underwent also lumbar 
puncture and CSF analysis of $A \beta_{42}$, t-Tau and p-Tau levels as well as a FDG-PET scan. Additionally, a conventional MRI scan was considered for visual analysis of atrophy in 60 individuals (i.e. 19 AD, 8 FTLD, 8 DLB, and 19 MCI).

All biomarker data were collected within 3 months from the baseline clinical visit. Each patient underwent a clinical and neuropsychological follow-up (27.48 \pm 10.43 months), that confirmed the diagnosis in dementia cases and in the MCI subjects who converted. Accordingly, MCI subjects were sub-grouped into converters and non-converters to AD.

\subsection{Study Design}

Firstly, two neurologists expert in dementia and blind to the previous clinical diagnosis reviewed all the included cases in order to formulate a possible diagnosis exclusively based on clinical information (i.e., case history, neurological examination and neuropsychological data), namely a "clinically-based classification". Sixteen patients out of 121 were excluded from the initial sample (i.e., cases without a complete diagnostic agreement between the two experts and/or not fulfilling clinical criteria for neurodegenerative dementia or for MCI condition [710,20,49]. Thus, the final cohort included 75 dementia patients (45 men and 30 women; mean age $=66.71 \pm 7.22$ years; Mini Mental State Examination $($ MMSE $)=18.41 \pm 5.26$; disease duration $=3.25 \pm 1.85$ ) either affected by AD, FTLD or DLB, and $30 \mathrm{MCI}$ subjects (15 men and 15 women; mean age $=68.24 \pm 6.8$ years; $M M S E=25.96 \pm 2.25)$. See Table 1 for details. Dementia was defined according to the clinical criteria of each main neurodegenerative dementia subtype [610,20]. MCI was defined as the presence of objective impairments at the neuropsychological evaluation in memory and/or other cognitive domains in the absence of functional impairment and dementia condition [49]. In addition, CSF A $\beta_{42}$, t-Tau and p-Tau levels as well as MRI and FDG-PET scans were evaluated according to the procedures reported below. In particular, CSF protein levels were considered as abnormal on the basis of established cut-off values [50]. Two 
expert neuroradiologists and two nuclear physician experts evaluated MRI and FDG-PET scans respectively, blind to clinical-neuropsychological information, in order to provide a final classification of the biomarker results (see following sections).

In a second step, in order to evaluate the additional value of the biomarkers, the same neurologists formulated a new diagnosis adding to the clinical data the information obtained by all the available biomarkers (i.e., CSF plus FDG-PET, and MRI), thus providing a "clinically/biomarker-based classification". The two diagnostic classifications (i.e., clinicallybased and clinically/biomarker-based) were then compared to the clinical diagnosis obtained with a follow-up of more than 2 years (follow-up diagnosis).

All subjects, or their informants/caregivers, gave informed consent, following detailed explanation, to the each experimental procedure, previously approved by the local Ethical Committee.

\subsection{CSF acquisition and analysis}

All patients underwent lumbar puncture in the L3-L4 or L4-L5 interspace after giving written informed consent, and following detailed explanation of the procedure. The procedure was always performed early in the morning. No serious adverse events were reported. CSF $(8-10 \mathrm{ml})$ was collected in sterile polypropylene tubes. Routine chemical parameters were determined; the remaining CSF was used to measure CSF $\mathrm{A} \beta_{42}$, t-Tau and p-Tau values. After centrifugation, CSF samples were stored at $-80^{\circ} \mathrm{C}$ until the analysis. Then, measurements of $\mathrm{A} \beta_{42}$, t-Tau and pTau levels were performed in the local laboratory (LABORAF, San Raffaele Hospital, Milan, Italy) by technicians blinded to the clinical diagnosis, using a commercially available ELISA kits (Innogenetics ${ }^{\circledR}$, Gent, Belgium), according to the manufacturer's instructions. Cut-off values for $\mathrm{AD}$ reported in the literature [50] were adopted, i.e. $\mathrm{A} \beta_{42} \geq 500 \mathrm{ng} / \mathrm{L} ; \mathrm{t}-\mathrm{Tau} \leq 350 \mathrm{ng} / \mathrm{L} ; \mathrm{p}-\mathrm{Tau} \leq$ $61 \mathrm{ng} / \mathrm{L}$. 


\subsection{MRI acquisition and analysis}

Brain MRI scans were acquired using a 3-Tesla Philips Achieva scanner (Philips Medical Systems, Best, NL) with an 8-channel head coil in a subgroup of 60 patients. Scanning session included T1-weighted volumetric MR scan $(220$ slices, TR/TE $=600 / 20 \mathrm{~ms}$, voxel size $\left.0.9 \times 0.9 \times 0.8 \mathrm{~mm}^{3}\right)$.

Each scan was evaluated and independently classified by two expert raters. Brain atrophy were considered according to the validated MRI patterns of the three main neurodegenerative dementia conditions (i.e., AD, DLB, and FTLD) [6-8,10,51]. Each scan received a label indicating whether it did not satisfy criteria for brain atrophy (i.e., Negative scan) or it was compatible with $\mathrm{AD}$ (AD-like pattern scan) or non-AD (non AD-like pattern scan) dementia condition.

\subsection{FDG-PET acquisition and analysis}

FDG-PET acquisitions were performed at the Nuclear Medicine Unit, San Raffaele Hospital (Milan, Italy), following standardized procedures [13]. All images were acquired with a Discovery STE (GE Medical Systems, Milwaukee, WI) multi-ring PET tomography (PET-CT) system (time interval between injection and scan start=45 minutes; scan duration 15 minutes). Images were reconstructed using an ordered subset expectation maximization (OSEM) algorithm. Each PET phase was corrected for attenuation with CT data of the corresponding phase. For each PET scan has been acquired either 35 or 47 (depending to the different scanner characteristics) transaxial tomographic slices of $4.25 \mathrm{~mm}$, re-oriented into the coronal and the sagittal planes. The emission images were then reconstructed using a filtered back-projection, using the software provided by the manufacturers. 
Image pre-processing and statistical analysis were performed according to the new procedure developed in our laboratory $[33,48]$. Each patient scan was then tested for relative "hypometabolism" by comparison with a large sample of FDG-PET scans from a database of normal controls on a voxel-by-voxel basis [33]. Proportional scaling was used to remove intersubject global variation in PET intensities. Additionally, voxel-wise comparisons were made using a within-brain comparison-specific explicit FDG mask in order to remove emission counts outside of the brain and to restrict subsequent analyses to within-brain voxels. The threshold was set at $\mathrm{p}=0.05$, FWE-corrected for multiple comparisons at the voxel level. Only clusters containing more than 100 voxels were deemed to be significant.

The FDG-PET hypometabolic patterns were classified by two nuclear physicians, blind to clinical-neuropsychological details, CSF and MRI results. Raters were asked to classify the SPM-t map as normal or abnormal. Namely, a normal SPM-t map should not reveal any significant hypometabolic pattern at a FWE-corrected threshold, either at the voxel or the cluster level. In case of abnormal findings, raters had to decide whether the hypometabolic pattern was suggestive of a specific neurodegenerative dementia subtype, according to a well-established literature $[6-8,12,33,35,52]$. So, each SPM-t map was classified as Negative, AD-like, DLB-like and FTLD-like following the criteria reported elsewhere [14].

\subsection{Statistical analysis}

Data were analyzed using the Statistical Package for Social Sciences software (SPSS V. 19.0). Socio-demographical differences between groups were assessed with Chi-square statistic for the dichotomous variables or Kruskal Wallis One-way analysis of variance by ranks for the continuous variables. 
First, we compared the clinically-based to the clinically/biomarker-based classification, in order to establish whether they presented discordances with respect to the reference follow-up diagnosis (Cohen's k analysis).

Cohen's k coefficient was also used to evaluate the inter-rater agreement between the experts' classifications for MRI and the FDG-PET imaging, resulting in an "almost perfect agreement" (k $>0.85$ ) in both cases. Thus, we selected a single set of classifications (i.e., the best one) for the subsequent analyses.

Then, we performed separate logistic regression analyses to evaluate the accuracy of each biomarker to correctly differentiate dementia subtypes. In particular, AD vs. non-AD (DLB+FTLD); FTLD vs. non-FTLD (DLB+AD) and DLB vs. non-DLB (FTLD+AD) comparisons were performed. This was crucial to establish the role of biomarkers in differentiating non-AD conditions. MRI was excluded from the latter two analyses (FTLD vs. non-FTLD and DLB vs. non-DLB) because of no specific DLB or FTLD classification was available for MRI scan.

In a further step, the significant regressors derived from the logistic regression analyses were joined in a logistic regression multivariate model. The regressors resulting from the multivariate analysis were considered as the most informative for the specific type of dementia diagnosis. Age and gender variables were inserted as covariates both for separate and multivariate logistic regression analyses, in order to verify whether their contribution to the model.

MCI who progressed to $\mathrm{AD}$ within the follow-up period (27.48 \pm 10.43 months) were compared to those who did not progress to $\mathrm{AD}$ and who remained stable, using Cox proportional hazard regression analysis. CSF, MRI and FDG-PET biomarkers were included in the multivariate analysis, in order to find those biomarkers significantly associated with progression to $\mathrm{AD}$. Each variable was entered in a stepwise forward manner. Thus, the final model was composed only by variables significantly predicting AD at the time of follow-up. Age and gender were inserted as covariates and follow-up time used as temporal variable of the model. Area 
under the curve (AUC), sensitivity (Sens), specificity (Spec), and positive and negative likelihood ratios (LR+ and LR-) were calculated for each biomarker. All statistical results were considered as significant with threshold $\mathrm{p} \leq 0.05$.

\section{RESULTS}

\subsection{Descriptive statistics}

No significant age difference was found among groups, except for DLB patients who were significantly older $(72.36 \pm 6.02$ years; $\mathrm{p}<0.001)$ than all the other groups (Table 1$)$. At the clinical follow-up, 10 out of $30 \mathrm{MCI}$ subjects converted to dementia. In particular, $8 \mathrm{MCI}$ converted to AD, 2 MCI converted to non-AD dementias (i.e., 1 FTLD and 1 DLB). MCI converter and non-converter groups did not show any significant demographic difference (Table $1)$.

\subsection{Comparison between clinically-based and clinically plus biomarker-based classifications}

Cohen's Kappa analysis demonstrated a significant but not perfect agreement between the clinically-based and clinically/biomarker-based classifications (Cohen's $\mathrm{K}=0.75 \mathrm{p}<0.001$ ), with a mismatch of $24 \%$ between them. Considering the follow-up diagnosis, the clinically/biomarker-based classification performed better than the clinically-based one. 


\subsection{Statistical analyses in dementia}

\subsubsection{AD vs. non- $A D$ comparison}

Among the CSF measures, the best performer for AD/non-AD classification was the ratio of $\mathrm{p}-\mathrm{Tau} / \mathrm{A} \beta_{42}(\operatorname{Exp} \beta=8.001 ; \mathrm{CI}=[2.55-25.15] ; \mathrm{p}<0.001 ; \mathrm{AUC}=0.81 ;$ Sens $=83 \% ; \mathrm{Spec}=64 \%$; $\mathrm{LR}+=2.3$; $\mathrm{LR}-=0.26)$. Although $\mathrm{A} \beta_{42}$ showed a good sensitivity $(85 \%)$, its specificity was poor (46\%). Since AD-related pathology may be present also in DLB patients, particularly in those with more severe cognitive impairment, we then excluded DLB cases from the non-AD dementia group. Thus, performing this new comparison analysis on AD vs. FTLD group, we found that, among the CSF biomarkers, $A \beta_{42}$ significant influenced the final regression model $(\operatorname{Exp} \beta=14.28$; $\mathrm{CI}=[3.49-58.54] ; \mathrm{p}<0.001 ; \mathrm{AUC}=0.78 ;$ Sens=85\%; Spec=71\%). Compared to $\mathrm{t}$-Tau, $\mathrm{p}$-Tau showed a better performance even if poorer than $\mathrm{p}$-Tau $/ \mathrm{A} \beta_{42}$ ratio $(\mathrm{AUC}=0.67$; Sens $=70 \%$; $\mathrm{Spec}=64 \%)$

Considering the two neuroimaging biomarkers, FDG-PET was the most accurate $(\operatorname{Exp} \beta=88$; $\mathrm{CI}=[18.17-426.13] ; \mathrm{p}<0.001 ; \mathrm{AUC}=0.90 ;$ Sens $=94 \% ; \mathrm{Spec}=86 \% ; \mathrm{LR}+=6.71 ; \mathrm{LR}-=0.07)$ whereas MRI showed both low sensitivity (46\%) and specificity (50\%) See Table 2. Notwithstanding the good performance of both FDG-PET and $\mathrm{p}$-Tau/A $\beta_{42}$ ratio, when entered together in a multivariate logistic regression model, only FDG-PET survived the statistical significance threshold.

Age and gender did not influence the results of the regression model.

\subsubsection{FTLD vs. non-FTLD and DLB vs. non-DLB comparisons}

The binomial logistic regression showed that FDG-PET was the most accurate biomarker to discriminate both FTLD from non-FTLD $(\operatorname{Exp} \beta=251.33 ; \mathrm{CI}=[24.17-2613-65] ; \mathrm{p}<0.001$; 
$\mathrm{AUC}=0.94 ;$ Sens $=93 \%$; Spec $=95 \%$; $\mathrm{LR}+=18.6 ; \mathrm{LR}-=0.07$ ) and DLB from non-DLB patients $(\operatorname{Exp} \beta=150 ; \mathrm{CI}=[15.17-1483.15] ; \mathrm{p}<0.001 ; \mathrm{AUC}=0.94 ; \mathrm{Sens}=71 \% ; \mathrm{Spec}=98 \% ; \mathrm{LR}+=35.5 ; \mathrm{LR}-$ $=0.3$ ). This result was confirmed also after entering age in the regression model as nuisance variable $(\operatorname{Exp} \beta=187.013 ; \mathrm{CI}=[12.30-2845.60] ; \mathrm{p}<0.000)$.

Among CSF biomarkers, the $A \beta_{42}$ value showed a good accuracy in distinguishing FTLD from non-FTLD patients $(\operatorname{Exp} \beta=0.07 ; \mathrm{CI}=[0.018-0.27] ; \mathrm{p}<0.001)$. Notably, FDG-PET and $\mathrm{A} \beta_{42}$ differently contributed to the correct discrimination of FTLD vs. non-FTLD patients (Figure 2). In particular, while a positive FDG-PET pattern for FTLD increased the likelihood to correctly classify FTLD cases, at the opposite, the positivity of $A \beta_{42}$ was in good agreement with the non-FTLD (AD or DLB) classification.

\subsection{Statistical analyses in MCI}

All biomarkers were considered in the multivariate Cox proportional hazards analysis comparing MCI converters and non converters to AD. FDG-PET was the only predictor of conversion in the final step-wise model $(\operatorname{Exp} \beta=8.62 ; \mathrm{CI}=[1.02-72.74] ; \mathrm{p}<0.05)$ (Figure 3$)$. The small number of patients prevented further analyses on MCI converters to other dementias (FTLD or DLB).

In the ROC analysis, both $\mathrm{t}-\mathrm{Tau} / \mathrm{A} \beta_{42}$ and $\mathrm{p}-\mathrm{Tau} / \mathrm{A} \beta_{42}$ ratios were informative in the prediction of conversion to $\mathrm{AD}$. Though the $\mathrm{p}-\mathrm{Tau} / \mathrm{A} \beta_{42}$ ratio showed a higher specificity compared to FDG-PET (96\% vs. 90\%), FDG-PET had a higher sensitivity ( $86 \%$ vs. 57\%). The lower FDG-PET specificity is due to the number of MCI with AD-like FDG-PET pattern who did not convert in the follow-up time, and thus considered by the ROC analysis as false positives. 


\section{DISCUSSION}

In this study, we evaluated the supportive role of CSF $A \beta_{42}$, t-Tau, $p$-Tau, conventional brain MRI and visual assessment of FDG-PET SPM t-map to early dementia diagnosis and MCI progression evaluation. In particular, we investigated the biomarkers' accuracy to recognize AD and non-AD dementias (DLB and FTLD), and to predict progression to dementia in MCI subjects.

The first key result of this study was a significant, but not perfect, agreement between the clinically-based and clinically/biomarker-based classifications. More specifically, only the clinically/biomarker-based classification resulted in a consistent diagnosis with reference to the follow-up diagnosis. This result indicates the significant supportive role of biomarkers in clinical settings, in order to improve clinicians' confidence for a correct diagnosis. Diagnostic errors have a number of undesirable implications. Both patient and caregivers may experience significant emotional stress and psychological damage related to a misdiagnosis of an incurable neurodegenerative condition. In addition, a wrong AD diagnosis may imply the administration of long-term pharmacological treatments (e.g., cholinesterase inhibitors) useless for non-AD conditions. It may also result in the misidentification of possible reversible conditions such as vitamin deficiency and depression.

As proved by the statistical analyses, the better performance of the clinically/biomarker-based classification is mainly due to the supportive role of the visual assessment of FDG-PET SPM tmaps. The semi-quantitative method here used provided indeed very high sensitivity (94\%) and specificity (86\%) in $\mathrm{AD}$ vs. non-AD recognition. FDG-PET was the best biomarker among those investigated, being able also to identify non-AD dementias (i.e., FTLD vs. non-FTLD: Sens $=93 \%$ and $\mathrm{Spec}=95 \%$; DLB vs. non-DLB: Sens $=71 \%$ and $\mathrm{Spec}=98 \%$ ). This was a crucial part of our analyses. While the majority of FDG-PET imaging studies in dementia investigated 
AD $[12,39,44]$, only a few explored AD vs. non-AD $[33,53]$. Nevertheless, FDG-PET has been included in the research diagnostic criteria of AD as well as non-AD dementia conditions [4$7,20,54]$.

Here we showed that the application of an optimized voxel-based method for FDG-PET scan evaluation was crucial to achieve a very high diagnostic accuracy. As previously validated by our group $[33,48]$, this tool had high sensitivity (96\%) and specificity $(84 \%)$ to recognize dementias, as well as to predict progression to dementia or reversion to normal cognition in MCI [14]. Its use guarantees not only higher diagnostic accuracy compared to the visual assessment of FDG-uptake, but also a higher level of confidence even for moderate-skilled FDGPET experts [33].

Among the CSF biomarkers, the $\mathrm{p}-\mathrm{Tau} / \mathrm{A} \beta_{42}$ ratio showed a good accuracy in distinguishing $\mathrm{AD}$ from non-AD as well as in predicting $\mathrm{MCI}$ conversion to $\mathrm{AD}$. These results agree with a recent meta-review [15] that reported $\mathrm{p}-\mathrm{Tau} / \mathrm{A} \beta_{42}$ ratio as the most accurate CSF measure for differentiating $\mathrm{AD}$ from controls and other dementias, and also for predicting $\mathrm{MCI}$ conversion to AD. In our study, the $\mathrm{p}-\mathrm{Tau} / \mathrm{A} \beta_{42}$ ratio, however, did not reach significance in the Cox regression analysis, indicating that CSF measures are less effective predictors of MCI conversion than imaging biomarkers $[55,56]$. Moreover, the $\mathrm{p}-\mathrm{Tau} / \mathrm{A} \beta_{42}$ ratio has limited value for the differential diagnosis of dementia, and it owns a low specificity possibly related to presence of patients with AD-related pathology in the non-AD group (i.e., DLB patients). The CSF profile of DLB, characterized by very low $A \beta_{42}$, with normal $A \beta_{40 / 42}$ ratio and moderate increase of $t-T a u$ and $p$ Tau levels, is in fact comparable to the AD CSF profile [57].

Qualitative evaluation of atrophy on MRI was the less informative biomarker both to support dementia classification and to predict MCI conversion to AD. Its low accuracy is probably related to different factors. Above all, the assessment of brain focal atrophy on MRI that is a fully operator-dependent procedure could be possibly biased with respect to semi-quantitative or quantitative procedures. Semi-quantitative or quantitative MRI approaches, however, are still 
unavailable as measurement at the single-subject level in the clinical setting, lacking of validated cut-off values. In addition, the diagnostic utility of medial temporal lobe atrophy recognition on MRI is very limited, as it is a common neuroradiological finding in other neurodegenerative neurological conditions, including frontotemporal dementia $[16,25,26,58]$ as well as in normal aging [28].

Statistical analyses in MCI confirmed that FDG-PET imaging is the most useful biomarker to predict further progression to dementia [14]. Noteworthy, FDG-PET SPM t-maps was able to identify, at the single-subject level, dysfunctional brain metabolic patterns typical of different dementia conditions. The high accuracy in identifying heterogeneous hypometabolic patterns, predictors of conversion to $\mathrm{AD}$ as well as to non-AD dementia, as also shown in a recent study [14], in this clinical context, confirms its high diagnostic value and suggests its role in avoiding multiple examinations in MCI subjects over months and years, which may lead to unnecessary delay in proper clinical management.

\section{CONCLUSIONS}

Considering the great overlap in clinical presentation among neurodegenerative disorders, diagnosis of dementia may be a difficult task in clinical practice, particularly in the early phase. Clinical and neuropsychological information alone may lead to a significant number of diagnostic errors and uncertainties, thus to unnecessary or inappropriate treatments. In this context, supportive findings from effective biomarkers have a great value in order to reach a more accurate early differential diagnosis. Here, in the routine clinical setting of our memory clinics, we have shown that the use of a validated and standardized method for semi-quantitative assessment of FDG-PET scan offers a solid support for diagnosis at the individual level with high accuracy and confidence. 
Future multi-centric studies, accounting for larger samples of dementia patients and MCI subjects, will increase the power of generalization of the present findings. The establishment of evidence-based standardized operation procedures is necessary for the correct use of biomarkers in clinical practice. 


\section{REFERENCES}

1. Prince M, Bryce R, Ferri C. World Alzheimer report 2011: the benefits of early diagnosis and intervention. Alzheimer's Disease International. 2011. 2013.

2. Geldmacher DS, Kirson NY, Birnbaum HG, Eapen S, Kantor E, Cummings AK, et al. Implications of early treatment among Medicaid patients with Alzheimer's disease. Alzheimer's Dement. Elsevier; 2014;10:214-24.

3. Gaugler JE, Ascher-Svanum H, Roth DL, Fafowora T, Siderowf A, Beach TG. Characteristics of patients misdiagnosed with alzheimer's disease and their medication use: an analysis of the NACC-UDS database. BMC Geriatr. BioMed Central Ltd; 2013;13:137.

4. Albert MS, DeKosky ST, Dickson D, Dubois B, Feldman HH, Fox NC, et al. The diagnosis of mild cognitive impairment due to Alzheimer's disease: recommendations from the National Institute on Aging-Alzheimer's Association workgroups on diagnostic guidelines for Alzheimer's disease. Alzheimers. Dement. [Internet]. Elsevier Ltd; 2011 [cited 2013 Sep 20];7:270-9.

Available from:

http://www.pubmedcentral.nih.gov/articlerender.fcgi?artid=3312027\&tool=pmcentrez\&renderty pe $=$ abstract

5. Sperling RA, Aisen PS, Beckett LA, Bennett DA, Craft S, Fagan AM, et al. Toward defining the preclinical stages of Alzheimer's disease: recommendations from the National Institute on Aging-Alzheimer's Association workgroups on diagnostic guidelines for Alzheimer's disease. Alzheimers. Dement. [Internet]. 2011 [cited 2013 Oct 17];7:280-92. Available from: http://www.pubmedcentral.nih.gov/articlerender.fcgi?artid=3220946\&tool=pmcentrez\&renderty pe $=$ abstract

6. McKeith IG, Dickson DW, Lowe J, Emre M, O’Brien JT, Feldman H, et al. Diagnosis and management of dementia with Lewy bodies: third report of the DLB Consortium. Neurology [Internet]. 2005 [cited 2013 Nov 7];65:1863-72. Available from:

http://www.neurology.org/content/65/12/1863.short

7. Rascovsky K, Hodges JR, Knopman D, Mendez MF, Kramer JH, Neuhaus J, et al. Sensitivity of revised diagnostic criteria for the behavioural variant of frontotemporal dementia. Brain [Internet]. 2011 [cited 2013 Nov 7];134:2456-77. Available from:

http://www.pubmedcentral.nih.gov/articlerender.fcgi?artid=3170532\&tool=pmcentrez\&renderty $\mathrm{pe}=\mathrm{abstract}$

8. Gorno-Tempini ML, Hillis AE, Weintraub S, Kertesz A, Mendez M, Cappa SF, et al. Classification of primary progressive aphasia and its variants. Neurology [Internet]. 2011 [cited 2013 Nov 7];76:1006-14. Available from:

http://www.pubmedcentral.nih.gov/articlerender.fcgi?artid=3059138\&tool=pmcentrez\&renderty $\mathrm{pe}=\mathrm{abstract}$

9. Armstrong MJ, Litvan I, Lang AE, Bak TH, Bhatia KP, Borroni B, et al. Criteria for the diagnosis of corticobasal degeneration. Neurology [Internet]. 2013 [cited 2013 Nov 13];80:496503. Available from: http://www.ncbi.nlm.nih.gov/pubmed/23359374 
10. Dubois B, Feldman HH, Jacova C, Hampel H, Molinuevo JL, Blennow K, et al. Advancing research diagnostic criteria for Alzheimer' s disease : the IWG-2 criteria. Lancet Neurol.

$2014 ; 13$.

11. Shaw LM, Korecka M, Clark CM, Lee VM-Y, Trojanowski JQ. Biomarkers of neurodegeneration for diagnosis and monitoring therapeutics. Nat. Rev. Drug Discov. [Internet]. 2007 [cited 2015 Jan 2];6:295-303. Available from:

http://www.ncbi.nlm.nih.gov/pubmed/17347655

12. Herholz K, Salmon E, Perani D, Baron JC, Holthoff V, Frölich L, et al. Discrimination between Alzheimer dementia and controls by automated analysis of multicenter FDG PET. Neuroimage [Internet]. 2002;17:302-16. Available from:

http://www.ncbi.nlm.nih.gov/pubmed/12482085

13. Anchisi D, Borroni B, Franceschi M, Kerrouche N, Kalbe E, Beuthien-Beumann B, et al. Heterogeneity of brain glucose metabolism in mild cognitive impairment and clinical progression to Alzheimer disease. Arch. Neurol. [Internet]. 2005;62:1728-33. Available from: http://www.ncbi.nlm.nih.gov/pubmed/16286547

14. Cerami C, Della Rosa PA, Magnani G, Santangelo R, Marcone A, Cappa SF, et al. Brain metabolic maps in Mild Cognitive Impairment predict heterogeneity of progression to dementia. NeuroImage Clin. Elsevier; 2014;

15. Ferreira D, Perestelo-Pérez L, Westman E, Wahlund L-O, Sarría A, Serrano-Aguilar P. Meta-Review of CSF Core Biomarkers in Alzheimer's Disease: The State-of-the-Art after the New Revised Diagnostic Criteria. Front. Aging Neurosci. [Internet]. 2014 [cited 2014 Oct 23];6:47. Available from:

http://www.pubmedcentral.nih.gov/articlerender.fcgi?artid=3970033\&tool=pmcentrez\&renderty $\mathrm{pe}=\mathrm{abstract}$

16. Frisoni GB, Fox NC, Jack CR, Scheltens P, Thompson PM. The clinical use of structural MRI in Alzheimer disease. Nat. Rev. Neurol. [Internet]. Nature Publishing Group; 2010 [cited 2014 Nov 13];6:67-77. Available from:

http://www.pubmedcentral.nih.gov/articlerender.fcgi?artid=2938772\&tool=pmcentrez\&renderty $\mathrm{pe}=\mathrm{abstract}$

17. Nordberg A, Carter SF, Rinne J, Drzezga A, Brooks DJ, Vandenberghe R, et al. A European multicentre PET study of fibrillar amyloid in Alzheimer's disease. Eur. J. Nucl. Med. Mol. Imaging [Internet]. 2013 [cited 2013 Nov 20];40:104-14. Available from:

http://www.pubmedcentral.nih.gov/articlerender.fcgi?artid=3510420\&tool=pmcentrez\&renderty $\mathrm{pe}=\mathrm{abstract}$

18. Lehmann M, Ghosh PM, Madison C, Laforce R, Corbetta-Rastelli C, Weiner MW, et al. Diverging patterns of amyloid deposition and hypometabolism in clinical variants of probable Alzheimer's disease. Brain [Internet]. 2013 [cited 2014 Jun 1];136:844-58. Available from: http://www.pubmedcentral.nih.gov/articlerender.fcgi?artid=3580269\&tool=pmcentrez\&renderty $\mathrm{pe}=\mathrm{abstract}$

19. Murray J, Tsui WH, Li Y, Mchugh P, Williams S, Pirraglia E, et al. FDG and Amyloid PET in Cognitively Normal Individuals at Risk for Late-Onset Alzheimer's Disease. Adv. Mol. imaging. 2014;4:15-26. 
20. McKhann GM, Knopman DS, Chertkow H, Hyman BT, Jack Jr CR, Kawas CH, et al. The diagnosis of dementia due to Alzheimer's disease: Recommendations from the National Institute on Aging-Alzheimer's Association workgroups on diagnostic guidelines for Alzheimer's disease. Alzheimer's Dement. Elsevier; 2011;7:263-9.

21. Coppi E, Ferrari L, Santangelo R, Caso F, Pinto P, Passerini G, et al. Further evidence about the crucial role of CSF biomarkers in diagnosis of posterior cortical atrophy. Neurol. Sci. Springer; 2014;35:785-7.

22. Santangelo R, Coppi E, Ferrari L, Bernasconi MP, Pinto P, Passerini G, et al. Cerebrospinal Fluid Biomarkers Can Play a Pivotal Role in the Diagnostic Work Up of Primary Progressive Aphasia. J. Alzheimer's Dis. IOS Press; 2015;43:1429-40.

23. Ferreira D, Rivero-Santana A, Perestelo-Pérez L, Westman E, Wahlund L-O, Sarría A, et al. Improving CSF biomarkers' performance for predicting progression from Mild Cognitive Impairment to Alzheimer's disease by considering different confounding factors: a metaanalysis. Front. Aging Neurosci. Frontiers Media SA; 2014;6.

24. Blennow K, Dubois B, Fagan AM, Lewczuk P, de Leon MJ, Hampel H. Clinical utility of cerebrospinal fluid biomarkers in the diagnosis of early Alzheimer's disease. Alzheimers. Dement. [Internet]. Elsevier Ltd; 2014;1-12. Available from: http://dx.doi.org/10.1016/j.jalz.2014.02.004

25. Jack CR, Dickson DW, Parisi JE, Xu YC, Cha RH, O’Brien PC, et al. Antemortem MRI findings correlate with hippocampal neuropathology in typical aging and dementia. Neurology. AAN Enterprises; 2002;58:750-7.

26. Barkhof F, Polvikoski TM, Van Straaten ECW, Kalaria RN, Sulkava R, Aronen HJ, et al. The significance of medial temporal lobe atrophy A postmortem MRI study in the very old. Neurology. AAN Enterprises; 2007;69:1521-7.

27. Hodges JR. Alzheimer's disease and the frontotemporal dementias: contributions to clinicopathological studies, diagnosis, and cognitive neuroscience. J. Alzheimer's Dis. IOS Press; 2013;33:S211-7.

28. Raji CA, Lopez OL, Kuller LH, Becker JT. Age , Alzheimer disease , and brain structure. Neurology. 2009;1899-905.

29. Perani D. Functional neuroimaging of cognition. Handb. Clin. Neurol. 2008;88:61.

30. Perani D. FDG-PET and amyloid-PET imaging: the diverging paths. Curr. Opin. Neurol. [Internet]. 2014 [cited 2014 Oct 16];27:405-13. Available from: http://www.ncbi.nlm.nih.gov/pubmed/24927239

31. Weiner MW, Veitch DP, Aisen PS, Beckett LA, Cairns NJ, Green RC, et al. The Alzheimer's Disease Neuroimaging Initiative: a review of papers published since its inception. Alzheimer's Dement. Elsevier; 2013;9:e111-94.

32. Sánchez-Juan P, Ghosh PM, Hagen J, Gesierich B, Henry M, Grinberg LT, et al. Practical utility of amyloid and FDG-PET in an academic dementia center. Neurology. AAN Enterprises; 2014;82:230-8. 
33. Perani D, Della Rosa PA, Cerami C, Gallivanone F, Fallanca F, Vanoli EG, et al. Validation of an optimized SPM procedure for FDG-PET in dementia diagnosis in a clinical setting.

NeuroImage. Clin. [Internet]. Elsevier B.V.; 2014 [cited 2014 Nov 28];6:445-54. Available from:

http://www.pubmedcentral.nih.gov/articlerender.fcgi?artid=4225527\&tool=pmcentrez\&renderty $\mathrm{pe}=\mathrm{abstract}$

34. Ossenkoppele R, Prins ND, Pijnenburg YAL, Lemstra AW, van der Flier WM, Adriaanse $\mathrm{SF}$, et al. Impact of molecular imaging on the diagnostic process in a memory clinic.

Alzheimer's Dement. Elsevier; 2013;9:414-21.

35. Cerami C, Crespi C, Della Rosa PA, Dodich A, Marcone A, Magnani G, et al. Brain changes within the visuo-spatial attentional network in posterior cortical atrophy. J. Alzheimer's Dis. IOS Press; 2015;43:385-95.

36. Frisoni GB, Bocchetta M, Chételat G, Rabinovici GD, de Leon MJ, Kaye J, et al. Imaging markers for Alzheimer disease: which vs how. Neurology [Internet]. 2013;81:487-500.

Available from: http://www.ncbi.nlm.nih.gov/pubmed/23897875

37. Frisoni GB, Perani D, Bastianello S, Bernardi G, Cappa SF, Trabucchi M. A roadmap to the use of biomarkers for the diagnosis of Alzheimer's disease in clinical practice: the Italian intersocietal consensus. Alzheimer's Dement.

38. Li Y, Rinne JO, Mosconi L, Pirraglia E, Rusinek H, DeSanti S, et al. Regional analysis of FDG and PIB-PET images in normal aging, mild cognitive impairment, and Alzheimer's disease. Eur. J. Nucl. Med. Mol. Imaging [Internet]. 2008 [cited 2013 Nov 15];35:2169-81. Available from:

http://www.pubmedcentral.nih.gov/articlerender.fcgi?artid=2693402\&tool=pmcentrez\&renderty $\mathrm{pe}=\mathrm{abstract}$

39. Edison P, Archer HA, Hinz R, Hammers A, Pavese N, Tai YF, et al. Amyloid, hypometabolism, and cognition in Alzheimer disease An [11C] PIB and [18F] FDG PET study. Neurology. AAN Enterprises; 2007;68:501-8.

40. Kasanuki K, Iseki E, Fujishiro H, Yamamoto R, Higashi S, Minegishi M, et al.

Neuropathological investigation of the hypometabolic regions on positron emission tomography with $[<\sup >18</$ sup $>$ F $]$ fluorodeoxyglucose in patients with dementia with Lewy bodies. J. Neurol. Sci. Elsevier; 2012;314:111-9.

41. Landau SM, Harvey D, Madison CM, Reiman EM, Foster NL, Aisen PS, et al. Comparing predictors of conversion and decline in mild cognitive impairment. Neurology [Internet]. 2010;75:230-8. Available from:

http://www.pubmedcentral.nih.gov/articlerender.fcgi?artid=2906178\&tool=pmcentrez\&renderty pe $=$ abstract

42. Prestia A, Caroli A, van der Flier WM, Ossenkoppele R, Van Berckel B, Barkhof F, et al. Prediction of dementia in MCI patients based on core diagnostic markers for Alzheimer disease. Neurology [Internet]. 2013;80:1048-56. Available from: http://www.ncbi.nlm.nih.gov/pubmed/23390179

43. Zhang D, Wang Y, Zhou L, Yuan H, Shen D. Multimodal classification of Alzheimer's disease and mild cognitive impairment. Neuroimage [Internet]. Elsevier Inc.; 2011 [cited 2013 
Sep 18];55:856-67. Available from:

http://www.pubmedcentral.nih.gov/articlerender.fcgi?artid=3057360\&tool=pmcentrez\&renderty $\mathrm{pe}=\mathrm{abstract}$

44. Morinaga A, Ono K, Ikeda T, Ikeda Y, Shima K, Noguchi-Shinohara M, et al. A comparison of the diagnostic sensitivity of MRI, CBF-SPECT, FDG-PET and cerebrospinal fluid biomarkers for detecting Alzheimer's disease in a memory clinic. Dement. Geriatr. Cogn. Disord. [Internet]. 2010 [cited 2013 Oct 9];30:285-92. Available from:

http://www.ncbi.nlm.nih.gov/pubmed/20861634

45. Shaffer JL, Petrella JR, Sheldon FC, Choudhury KR, Calhoun VD, Coleman RE, et al. Predicting cognitive decline in subjects at risk for Alzheimer disease by using combined cerebrospinal fluid, MR imaging, and PET biomarkers. Radiology. Radiological Society of North America, Inc.; 2013;266:583-91.

46. Choo IH, Ni R, Schöll M, Wall A, Almkvist O, Nordberg A. Combination of (18)F-FDG PET and cerebrospinal fluid biomarkers as a better predictor of the progression to Alzheimer's disease in mild cognitive impairment patients. J. Alzheimers. Dis. [Internet]. 2013 [cited 2013 Oct 9];33:929-39. Available from: http://www.ncbi.nlm.nih.gov/pubmed/23047371

47. Alexopoulos P, Kriett L, Haller B, Klupp E, Gray K, Grimmer T, et al. Limited agreement between biomarkers of neuronal injury at different stages of Alzheimer's disease. Alzheimer's Dement. Elsevier; 2014;10:684-9.

48. Della Rosa PA, Cerami C, Gallivanone F, Prestia A, Caroli A, Castiglioni I, et al. A Standardized [18F]-FDG-PET Template for Spatial Normalization in Statistical Parametric Mapping of Dementia. Neuroinformatics. Springer; 2014;12:575-93.

49. Petersen RC, Roberts RO, Knopman DS, Boeve BF, Geda YE, Ivnik RJ, et al. Mild cognitive impairment: ten years later. Arch. Neurol. American Medical Association; 2009;66:1447-55.

50. Tapiola T, Alafuzoff I, Herukka S-K, Parkkinen L, Hartikainen P, Soininen H, et al. Cerebrospinal fluid \{beta\}-amyloid 42 and tau proteins as biomarkers of Alzheimer-type pathologic changes in the brain. Arch. Neurol. [Internet]. American Medical Association; 2009 [cited 2013 Nov 3];66:382-9. Available from:

http://archneur.jamanetwork.com/article.aspx?articleid=796677

51. Jack Jr CR, Albert MS, Knopman DS, McKhann GM, Sperling RA, Carrillo MC, et al. Introduction to the recommendations from the National Institute on Aging-Alzheimer's Association workgroups on diagnostic guidelines for Alzheimer's disease. Alzheimer's Dement. Elsevier; 2011;7:257-62.

52. Salmon E, Garraux G, Delbeuck X, Collette F, Kalbe E, Zuendorf G, et al. Predominant ventromedial frontopolar metabolic impairment in frontotemporal dementia. Neuroimage [Internet]. 2003 [cited 2013 Nov 13];20:435-40. Available from:

http://www.sciencedirect.com/science/article/pii/S105381190300346X

53. Foster NL, Heidebrink JL, Clark CM, Jagust WJ, Arnold SE, Barbas NR, et al. FDG-PET improves accuracy in distinguishing frontotemporal dementia and Alzheimer's disease. Brain [Internet]. 2007 [cited 2013 Nov 19];130:2616-35. Available from:

http://www.ncbi.nlm.nih.gov/pubmed/17704526 
54. Gorno-Tempini ML, Dronkers NF, Rankin KP, Ogar JM, Phengrasamy L, Rosen HJ, et al. Cognition and anatomy in three variants of primary progressive aphasia. Ann. Neurol. [Internet]. 2004 [cited 2013 Nov 7];55:335-46. Available from:

http://www.pubmedcentral.nih.gov/articlerender.fcgi?artid=2362399\&tool=pmcentrez\&renderty $\mathrm{pe}=\mathrm{abstract}$

55. Fellgiebel A, Scheurich A, Bartenstein P, Müller MJ. FDG-PET and CSF phospho-tau for prediction of cognitive decline in mild cognitive impairment. Psychiatry Res. Neuroimaging. Elsevier; 2007;155:167-71.

56. Walhovd KB, Fjell a M, Brewer J, McEvoy LK, Fennema-Notestine C, Hagler DJ, et al. Combining MR imaging, positron-emission tomography, and CSF biomarkers in the diagnosis and prognosis of Alzheimer disease. AJNR. Am. J. Neuroradiol. [Internet]. 2010 [cited 2013 Oct 20];31:347-54. Available from:

http://www.pubmedcentral.nih.gov/articlerender.fcgi?artid=2821467\&tool=pmcentrez\&renderty pe $=$ abstract

57. Kaerst L, Kuhlmann A, Wedekind D, Stoeck K, Lange P, Zerr I. Using cerebrospinal fluid marker profiles in clinical diagnosis of dementia with Lewy bodies, Parkinson's disease, and Alzheimer's disease. J. Alzheimers. Dis. [Internet]. 2014 [cited 2015 Jan 2];38:63-73. Available from: http://www.ncbi.nlm.nih.gov/pubmed/23948928

58. Van de Pol LA, Hensel A, Barkhof F, Gertz HJ, Scheltens P, van der Flier WM. Hippocampal atrophy in Alzheimer disease: age matters. Neurology [Internet]. 2006 [cited 2013 Nov 20];66:236-8. Available from: http://www.ncbi.nlm.nih.gov/pubmed/16434661 


\section{LEGEND:}

Figure 1. Best models for each dementia subgroup obtained performing ROC curve and Logistic regression analyses for A) AD vs. non-AD comparison; B) FTLD vs. non-FTLD comparison and C) DLB vs- non-DLB comparison. In the horizontal axis, the specificity values and in the vertical axis the sensitivity values. Ref $=$ Reference line; FDG-PET $=$ fluorodeoxyglucose positron emission tomography; $\mathrm{p}-\mathrm{Tau} / \mathrm{A} \beta_{42}=$ phosphorylated $\mathrm{Tau} / \mathrm{A} \beta_{42}$ ratio.

Figure 2. Positive and negative likelihood ratio (LR+ and LR-) for correct classification between patients with Alzheimer's disease (AD), frontotemporal lobar degeneration (FTLD) and dementia with Lewy body (DLB). A positive LR greater than 5 indicates that the biomarker positive classification is more probably associated with the disease occurrence. A negative LR below 0.2 indicates a relevant association between negative biomarker classification and the absence of dementia condition. $\mathrm{p}$-Tau/A $\beta_{42}=$ phosphorylated Tau / $\mathrm{A} \beta_{42}$ ratio; $\mathrm{t}$-Tau $/ \mathrm{A} \beta_{42}=$ total $\mathrm{Tau} / \mathrm{A} \beta_{42}$ ratio; $\mathrm{p}-\mathrm{Tau}=$ phosphorylated $\mathrm{Tau} ; \mathrm{t}-\mathrm{Tau}=$ total $\mathrm{Tau} ; \mathrm{MRI}=$ magnetic resonance imaging; FDG-PET = fluorodeoxyglucose positron emission tomography.

Figure 3. Survival curves of MCI converters vs. MCI not converters within the follow-up time according to the FDG-PET pattern at the baseline. None of the MCI subjects with negative FDGPET pattern $(n=8)$ converted to AD (blue line); one of the MCI subjects with FDG-PET AD positive pattern converted to non-AD dementia (grey line); six MCI subjects classified as AD positive with FDG-PET converted to AD (green line) showing the higher risk (lower level of survival). 
Table 1. Demographical and clinical characteristics of the sample. Values are mean (standard deviation). * = significant difference between DLB and all other groups; ${ }^{\#}=$ significant differences between MCI-converter and MCI-non converter and the other groups. AD = Alzheimer's Disease; DLB = Dementia with Lewy Bodies; FTLD = Frontotemporal Lobar Degeneration; MCI = Mild Cognitive Impairment; MMSE = Mini Mental State Examination.

\begin{tabular}{|l|l|l|l|l|l|l|}
\hline & AD (n=47) & FTLD (n=14) & DLB (n=14) & MCI-converter (n=10) & MCI-non converter (n=20) & P value \\
\hline Age & $66(6.8)$ & $65(7.3)$ & $72(6)$ & $69(5.5)$ & $68(7.6)$ \\
\hline Gender (m/f) & $26 / 21$ & $8 / 6$ & $11 / 3$ & $7 / 4$ & $8 / 11$ & $40.05 *$ \\
\hline Disease Duration & $39(24)$ & $32(19)$ & $42(22)$ & $28(10.9)$ & N.S. \\
\hline MMSE & $18(4.5)$ & $20(7.1)$ & $16(5.2)$ & $26(1.8)$ & $26(2.5)$ \\
\hline
\end{tabular}


Table 2. ROC analyses including sensitivity and specificity of each biomarker. In bold AD = Alzheimer's disease; FTLD = frontotemporal lobar degeneration; DLB = dementia with Lewy body; $\mathrm{AUC}=$ area under the curve; Sens = sensitivity; Spec $=$ specificity; $\mathrm{t}=$ Tau $=$ total Tau; $\mathrm{p}-\mathrm{Tau}=$ phosphorylated Tau; MRI = magnetic resonance imaging; FDG-PET = fluorodeoxyglucose positron emission tomography.

\begin{tabular}{|c|c|c|c|c|c|c|c|c|c|}
\hline & \multicolumn{3}{|c|}{$A D$ vs. non- $A D$ Comparison } & \multicolumn{3}{|c|}{ FTLD vs. non-FTLD Comparison } & \multicolumn{3}{|c|}{ DLB vs. non-DLB Comparison } \\
\hline & ROC AUC & Sens $\%$ & Spec \% & ROC AUC & Sens \% & Spec \% & ROC AUC & Sens $\%$ & Spec \% \\
\hline$A \beta_{42}$ & 0.64 & $85 \%$ & $43 \%$ & 0.22 & $29 \%$ & $15 \%$ & 0.57 & $86 \%$ & $72 \%$ \\
\hline$t$-Tau & 0.62 & $38 \%$ & $86 \%$ & 0.45 & $21 \%$ & $69 \%$ & 0.36 & $7.1 \%$ & $34 \%$ \\
\hline$p$-Tau & 0.67 & $70 \%$ & $64 \%$ & 0.37 & $36 \%$ & $38 \%$ & 0.37 & $36 \%$ & $62 \%$ \\
\hline$t-T a u / A \beta_{42}$ & 0.80 & $79 \%$ & $68 \%$ & 0.20 & $36 \%$ & $23 \%$ & 0.31 & $43 \%$ & $77 \%$ \\
\hline$p-T a u / A \beta_{42}$ & $\mathbf{0 . 8 1}$ & $83 \%$ & $64 \%$ & 0.23 & $43 \%$ & $16 \%$ & 0.32 & $57 \%$ & $84 \%$ \\
\hline$M R I$ & 0.52 & $46 \%$ & $50 \%$ & - & - & - & - & - & - \\
\hline$F D G-P E T$ & 0.90 & $94 \%$ & $86 \%$ & \begin{tabular}{|l|}
0.94 \\
\end{tabular} & $93 \%$ & $95 \%$ & 0.94 & $71 \%$ & $98 \%$ \\
\hline
\end{tabular}




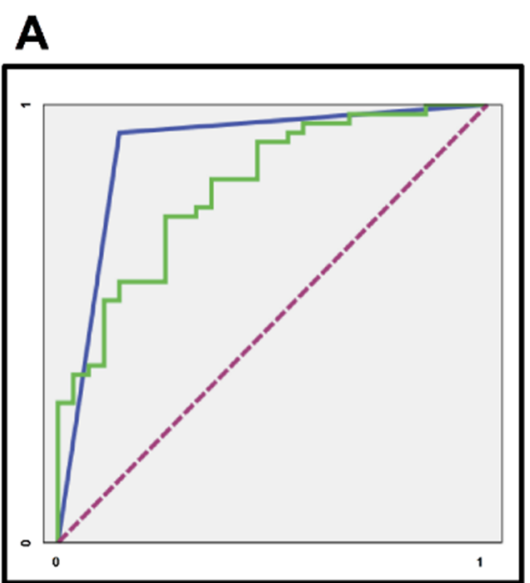

$A D$ vs. non-AD

Best Comparison Model

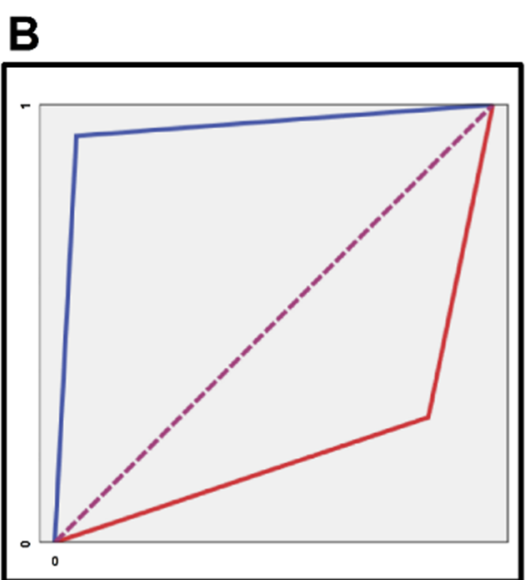

FTLD vs. non-FTLD Best Comparison Model

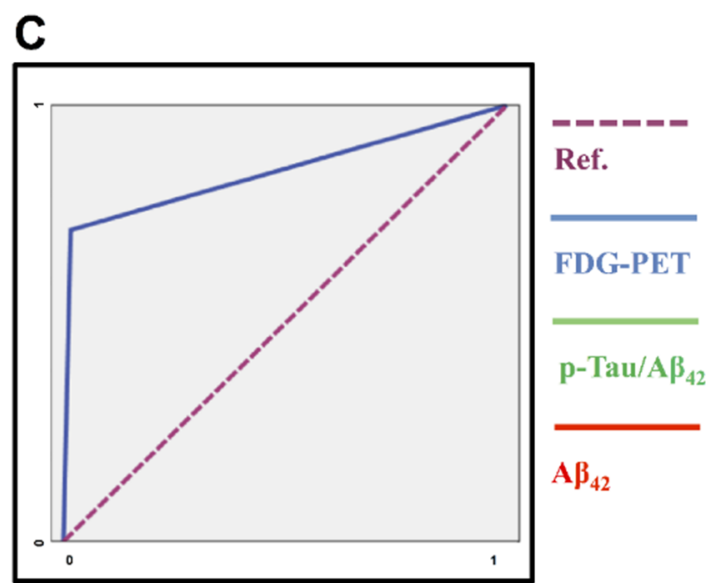

DLB vs. non-DLB Best Comparison Model 


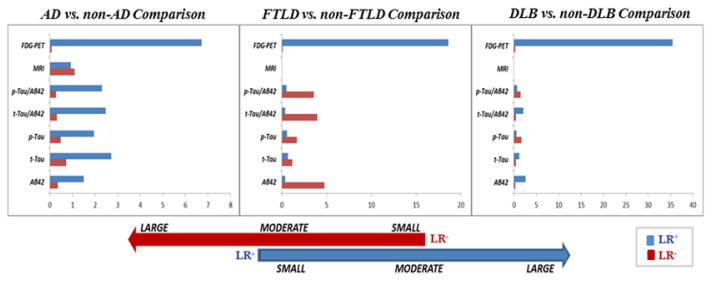




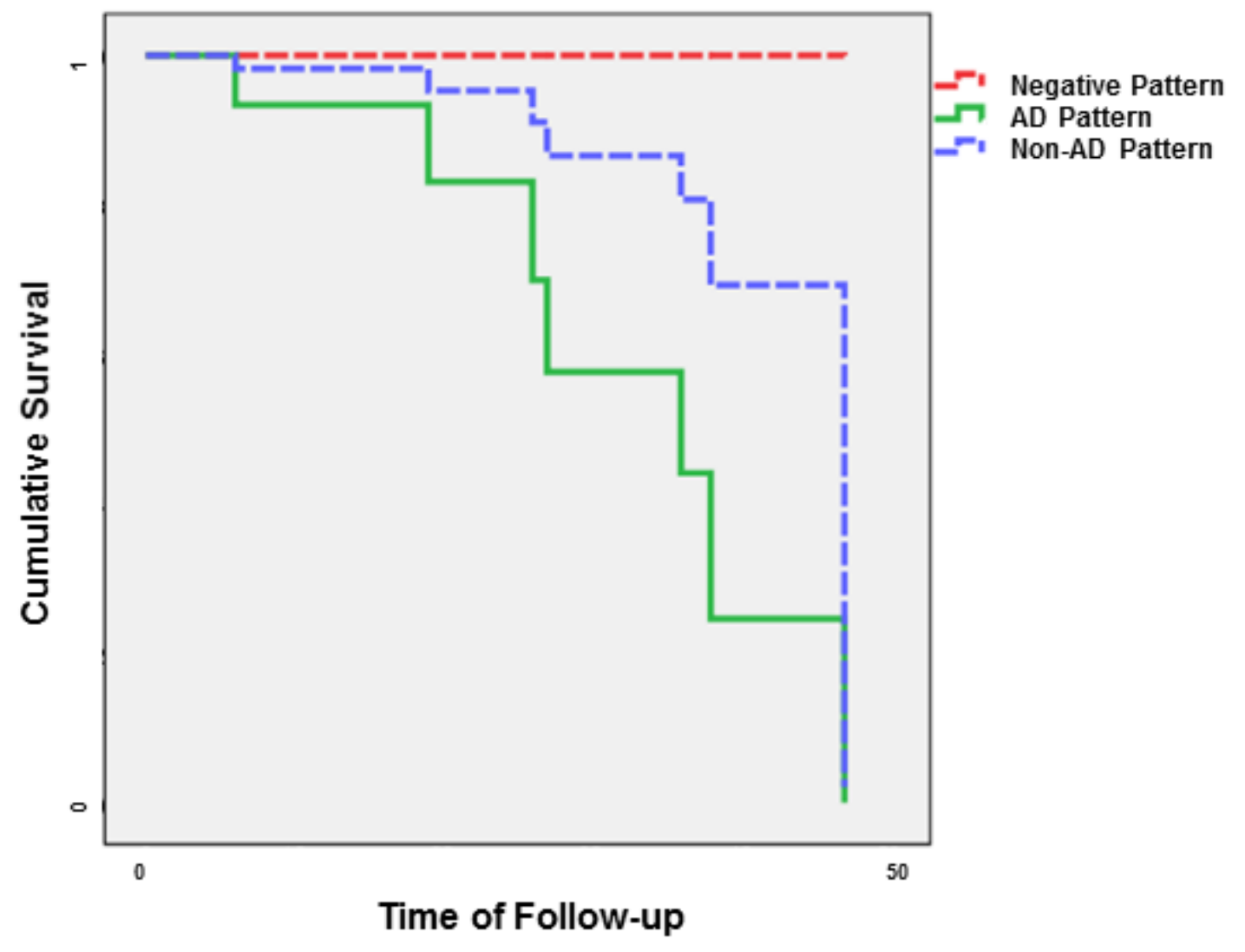

